\title{
Isophase Transitions of Cellulose - A Short Review
}

\author{
By Michael Ioelovich*
}

\begin{abstract}
Cellulose has a complicated supramolecular structure consisting of nanofibrils, which are built of ordered crystallites and low ordered noncrystalline domains. In this critical review, isophase temperature transitions in noncrystalline domains of cellulose were described and discussed. It has been shown that due to structural heterogeneity the noncrystalline domains have three isophase temperature transitions, where the $\alpha 1$ and $\alpha 2$ transitions are caused by the occurrence of segmental mobility in dense mesomorphous and medium packed amorphous clusters, respectively; whereas the $\beta$ transition is related to the mobility of small segments in loose packed amorphous clusters, which probably are located on the outer surface of nanofibrils. Under the action of water and other plasticizers all three isophase transitions are shifted to lower temperatures.
\end{abstract}

Keywords: Cellulose, Noncrystalline domains, Relaxation transitions, Mechanism.

\section{Introduction}

As is known, the properties of polymer materials are changed gradually in a certain temperature range until achieving the critical temperature or temperatures, at which an abrupt alteration of various characteristics is observed. This phenomenon can signify the so called isophase transition connected with structural reorganization within the same, e.g. amorphous, phase (Boyer, 1967; Shen et al., 1970). Typical examples of isophase transitions are glass or $\boldsymbol{\alpha}$ relaxation transitions caused by a development of segmental mobility, and secondary or $\boldsymbol{\beta}$ and $\boldsymbol{\gamma}$ relaxation transitions, which are connected with change of mobility of some units or groups in an amorphous phase. The isophase transitions appear as inflection points on temperature dependences of volume, deformation, modulus and other mechanical characteristics; jumps of thermal expansion coefficient and heat capacity; extremum of dynamic properties; but these transitions do not change the thermodynamic characteristics of polymers.

Linear amorphous polymers with a simple structure typically have one glass temperature $\left(\mathrm{T}_{\mathrm{g}}\right)$. For example, natural rubber transits from glass into viscoelastic state at $200 \mathrm{~K}$. However, polymers with complicated structure can have multiple isophase transitions. One such polymer, polyethylene, has three glass transitions: $153 \mathrm{~K}$ for completely amorphous domains, $240 \mathrm{~K}$ for mesomorphous domains on, and at $200 \mathrm{~K}$ for intermediate amorphous domains (Bartenev et at., 1981; Fakirov et al., 2000).

\footnotetext{
${ }^{*}$ Chief Chemist, DEsigner Energy Ltd, Israel.
} 
Cellulose is the most abundant natural polysaccharide, which can be used as a renewable source for the production of diverse biomaterials, biochemicals and biofuels (Klemm et al., 2005). Since the processing and use of cellulose can be carried out over a wide temperature range, it is important to study the isophase transitions of this polymer. Cellulose is a semicrystalline polysaccharide composed of glucopyranose units (GPU) linked by $\beta-1,4-$ glycosidic bonds. The linear macromolecules of cellulose form strong crystallites and weak non-crystalline (amorphous) domains by means of hydrogen bonds (Ioelovich, 2014). The relaxation state of strong cellulose crystallites does not change up to the melting point, which is above temperature of thermal decomposition (Ioelovich, 2016). On the contrary, the weak non-crystalline domains show isophase transitions of various types in a wide temperature range below temperature of thermal decomposition (Kaimin et al., 1979). Cellulose can be of various modifications and crystallinities, including natural, mercerized, regenerated, microcrystalline, nanocrystalline, amorphized, etc. Therefore, such a complex polymer as cellulose has multiple isophase transitions connected with abrupt changes in the relaxation state of non-crystalline domains, which are discussed in this article.

\section{Isophase Transitions of Cellulose}

Currently it is supposed that primary $\boldsymbol{\alpha}_{1}$ glass transition of cellulose is located at high-temperatures, above $473 \mathrm{~K}\left(\mathrm{~T}_{\mathrm{g} 1}\right)$ (Back et al., 1969; Kaimin et al., 1979). However, a direct measurement of the exact value of $T_{g 1}$ is difficult due to the beginning of thermal decomposition of cellulose. To evaluate $\mathrm{T}_{\mathrm{g} 1}$, indirect methods were used. One such method was the determination of glass temperatures $\left(\mathrm{T}_{\mathrm{g}}\right)$ for plasticized cellulose samples at temperatures below temperature of thermal decomposition; the obtained glass temperatures were extrapolated to the value corresponding to zero content of plasticizer (Ioelovich et al., 1979; Ioelovich, 2016; Kargin et al., 1960), as it is shown in Figure 1.

Figure 1. Illustration of Dependence of Glass Transition Temperature on Content of Plasticizer

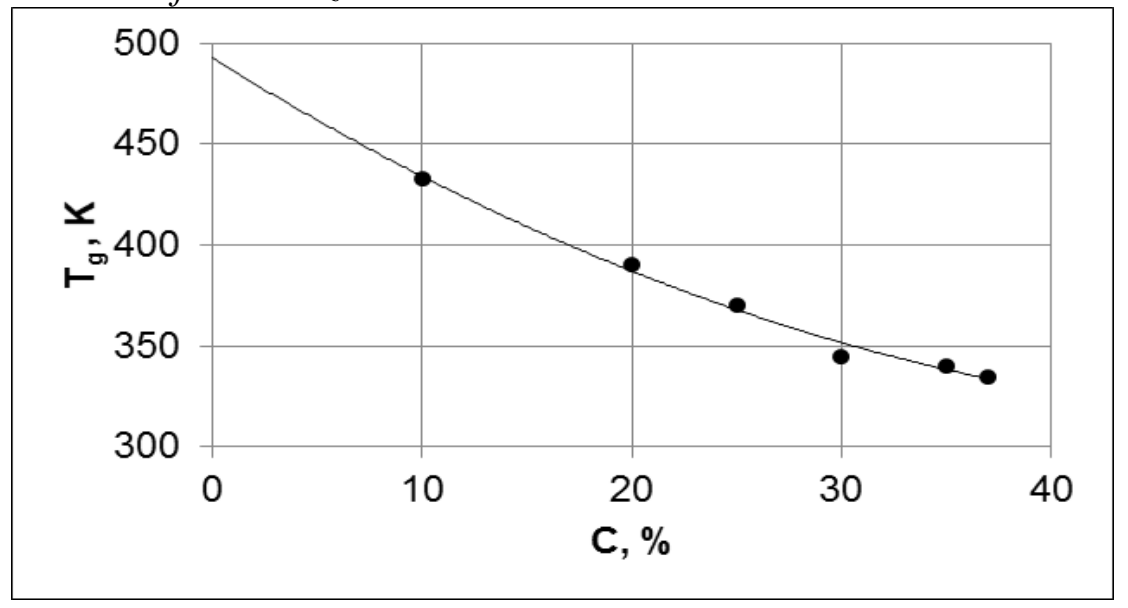


Another indirect method was the determination of glass temperatures $\left(T_{g}\right)$ of oligosaccharides with various degree of polymerization (DP) (Alftha et al., 1973 ) with the subsequent extrapolation of linear dependence $T_{g}=\left(D^{-1}\right)$ to zero value of $\mathrm{DP}^{-1}$ (Figure 2).

Figure 2. Illustration of Dependence of Glass Transition Temperature $\left(T_{g}\right)$ on Degree of Polymerization (DP) of Cellulose Oligomers

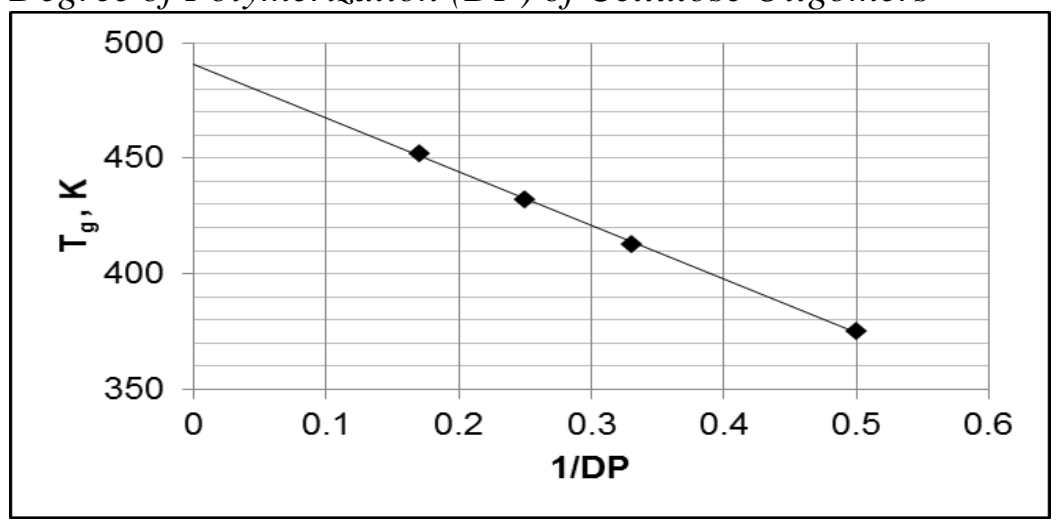

The third method is a calculation based on the ratio: $\mathrm{T}_{\mathrm{g}} / \mathrm{T}_{\mathrm{m}}=0.66$ (Boyer, 1967; Van Krevelen, 1972); where the melting point, $\mathrm{T}_{\mathrm{m}} \approx 720-770 \mathrm{~K}$, was indirectly estimated from an experiment of flash fusion of cellulose crystallites by a laser beam (Nordin et al., 1973). As a result of indirect evaluations, the following primary glass temperature of cellulose was obtained: $\mathrm{T}_{\mathrm{g} 1} \approx 490-500$ $\mathrm{K}$ (Table 1). The apparent activation energy of this transition was above 200 $\mathrm{kJ} / \mathrm{mole}$ (Manabe et al., 1986), which is typical for glass transition.

Table 1. Isophase Relaxation Transitions of Cellulose

\begin{tabular}{|c|c|c|c|}
\hline$* \mathbf{T}_{\mathrm{t}}, \mathbf{K}$ & Interpretation & Methods & References \\
\hline $490-500$ & $\begin{array}{l}\text { Primary } \boldsymbol{\alpha}_{1} \text { glass } \\
\text { transition }\end{array}$ & Indirect methods & $\begin{array}{l}\text { Alftha et al., 1973; } \\
\text { Ioelovich et al., 1979; } \\
\text { Kargin et al., } 1960 .\end{array}$ \\
\hline $390-410$ & $\begin{array}{c}\text { Secondary } \boldsymbol{\alpha}_{2} \text { glass } \\
\text { transition }\end{array}$ & $\begin{array}{l}\text { Dynamic methods, } \\
\text { dilatometry, } \\
\text { thermomechanics, } \\
\text { calorimetry }\end{array}$ & $\begin{array}{l}\text { Back et al., 1969; } \\
\text { Fainberg et al., 1967; } \\
\text { Kaimin et al., 1973; } \\
\text { Klason et al., 1976; } \\
\text { Manabe et. al., } 1986 .\end{array}$ \\
\hline $280-300$ & $\begin{array}{c}\boldsymbol{\beta} \text { transition of } \\
\text { small segments }\end{array}$ & $\begin{array}{l}\text { Dilatometry, } \\
\text { thermomechanics, } \\
\text { dynamic dielectric } \\
\text { method, DSC, EPR }\end{array}$ & $\begin{array}{l}\text { Aziz et al., 1973; } \\
\text { Kaimin et al., 1973; } \\
\text { Klason et al., 1976; } \\
\text { Mochalov et al., 1982; } \\
\text { Plotnikov et al., 1977; } \\
\text { Ramiah et al.,1965; } \\
\text { Roig et al., 2011. }\end{array}$ \\
\hline $180-200$ & $\begin{array}{l}\gamma \text { transition of } \\
\text { hydroxymethyl } \\
\text { groups }\end{array}$ & $\begin{array}{c}\text { Dynamic dielectric } \\
\text { methods }\end{array}$ & $\begin{array}{l}\text { Borisova et al., 1979; } \\
\text { Michailov et al., 1967; } \\
\text { Roig et al., 2011; } \\
\text { Zelenev et al., 1972. }\end{array}$ \\
\hline
\end{tabular}

$* \mathbf{T}_{\mathbf{t}}$ is the temperature of isophase transition. 
Below $\mathrm{T}_{\mathrm{g} 1}$, at the temperatures $390-410 \mathrm{~K}$, the $\boldsymbol{\alpha}_{2}$-relaxation transition was discovered by various experimental methods (Table 1): sharp decrease of dynamic modulus (Back et al., 1969), peak of mechanical absorption (Manabe et. al., 1986), sharp increase of deformation (Kaimin et al., 1973), jumps of heat capacity (Fainberg et al., 1967) and thermal expansion coefficient (Kaimin et al., 1973; Klason et al., 1976) (see for example Figures 3 and 4). The apparent activation energy of the $\boldsymbol{\alpha}_{2}$-transition was above $100 \mathrm{~kJ} / \mathrm{mole}$, which is higher than for the $\boldsymbol{\beta}$ transition. Therefore, most researchers attribute this transition to a secondary glass transition (Fainberg et al., 1967; Kaimin et al., 1973; Kaimin et al., 1979; Klason et al., 1976).

The third relaxation transition was detected at the temperatures 280-300 K by methods of thermomechanics (Kaimin et al., 1973), calorimetry (Mochalov et al., 1982), electron paramagnetic resonance (Plotnikov et al., 1977), dynamic dielectric method (Roig et al., 201), linear and volume dilatometry (Aziz et al., 1973; Kaimin et al., 1973; Klason et al., 1976; Ramiah et al., 1965) (see for example Figures 3, and 4). However, changes of various properties at a transition temperature were relatively small. The energy of activation of this transition was about $80 \mathrm{~kJ} / \mathrm{mole}$ (Borisova et al., 1979), which is typical for $\boldsymbol{\beta}$ transition.

Figure 3. Linear Dilatometry of Dry Film of Regenerated Cellulose

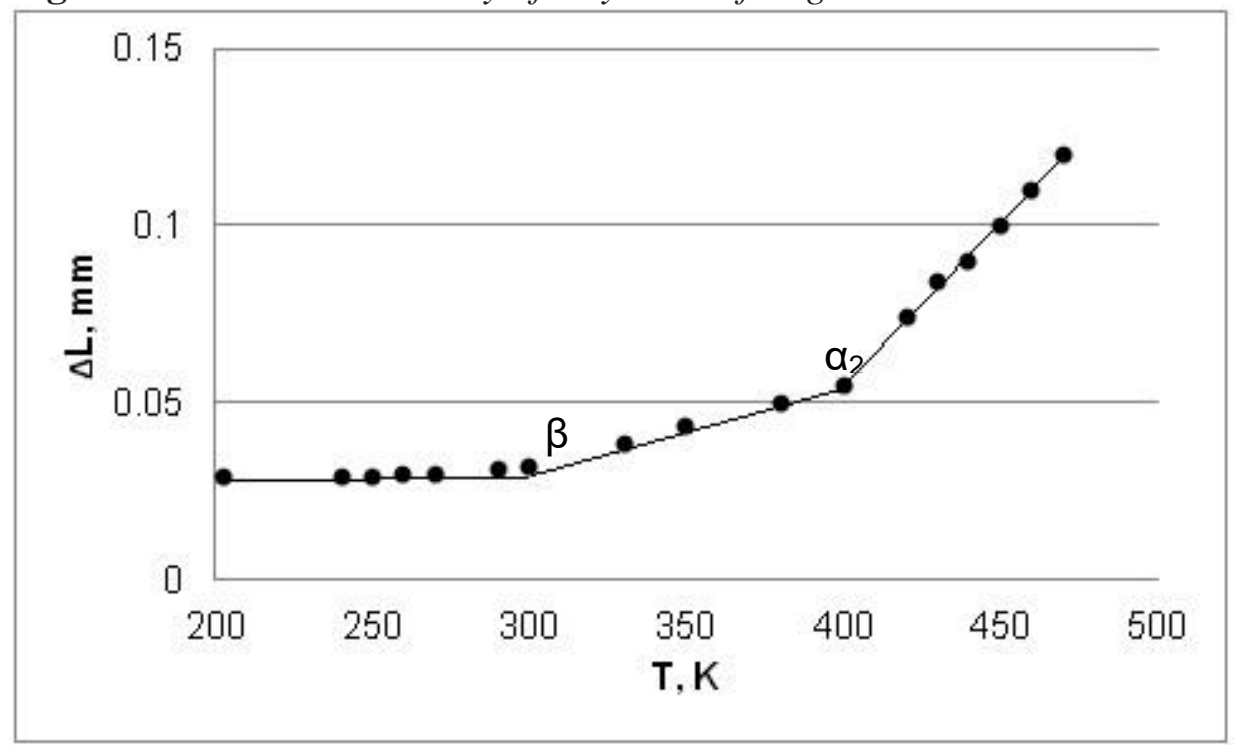


Figure 4. Temperature Dependence of Volume Expansion Coefficient for Dry Samples of Cotton Cellulose (CC) and Regenerated Cellulose (RC) with Different Crystallinity Degree $(X)$

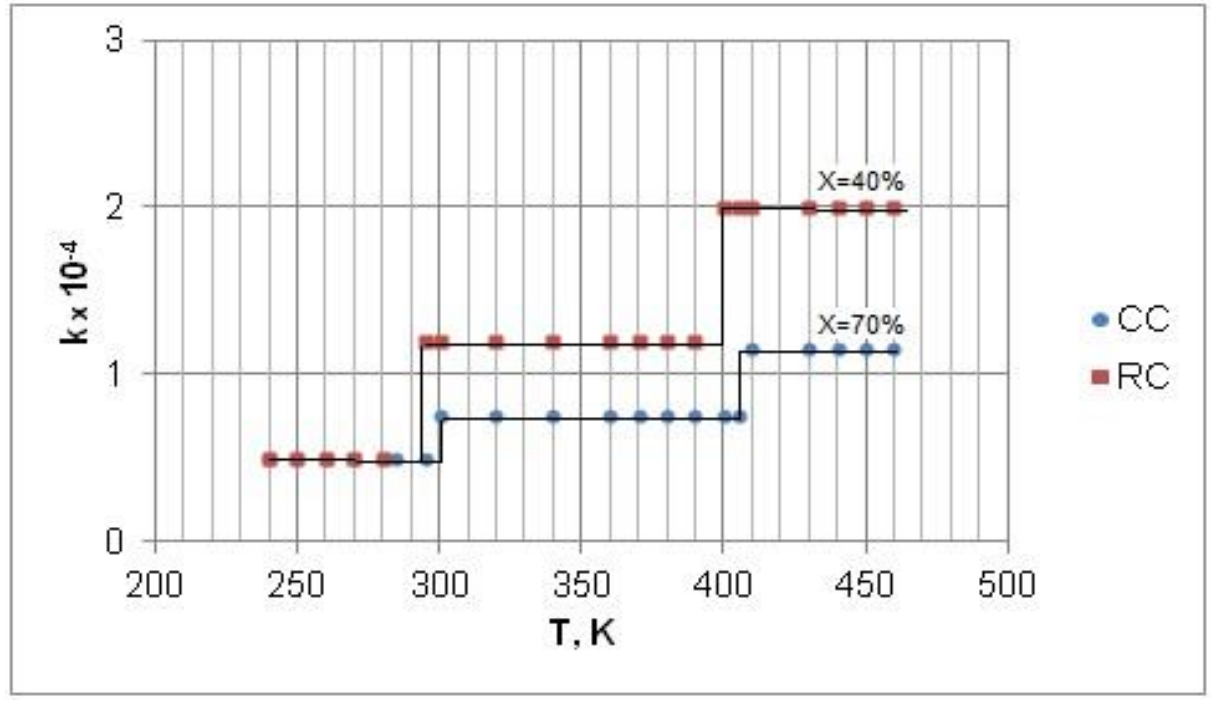

The fourth relaxation transition was found at 180-200 K using a dynamic method of dialectical absorption at low temperatures (Borisova et al., 1979; Michailov et al., 1967; Roig et al., 2011; Zelenev et al., 1972). It has been proven that it is a $\gamma$ transition with an activation energy of $40-50 \mathrm{~kJ} / \mathrm{mole}$, which is caused by the mobility of hydroxymethyl groups in non-crystalline domains.

With decreasing in the crystallinity degree, the intensity of $\boldsymbol{\alpha}, \boldsymbol{\beta}$ and $\boldsymbol{\gamma}$ transitions increases; thus all these transitions are related to non-crystalline domains of cellulose.

In contrast to elastomers, cellulose materials even above glass transition temperatures show a high modulus (1-3 GPa), low deformation (a few percents), as well as small changes of some other properties at transition temperatures. Therefore, to discover isophase temperature transitions in this polymer, a special high-precise research method should be used.

\section{Structural Organization of Noncrystalline Domains}

To explain multiple isophase transitions of cellulose the structural organization of non-crystalline domains (NCD) of this polymer should be studied in detail. However, these investigations are hampered by the presence of highly ordered crystalline domains, and by the lack of reliable methods for the study of noncrystalline components. For instance, various NMR methods gave a little information about the structure of NCD (Wickholm et al., 2001).

To study the structure of NCD by a method of X-ray diffraction, samples of amorphous cellulose are required. These samples can be prepared, for example, by ball-milling of dry cellulose or saponification of cellulose acetate in non-water alkali solutions (Paes et al., 2010; Togawa et al., 2007). The 
typical X-ray scattering of amorphous cellulose samples has a wide peak with its maximum at $2 \theta \approx 20^{\circ}$ (Figure 5).

Figure 5. X-ray Scattering of Amorphized Cellulose

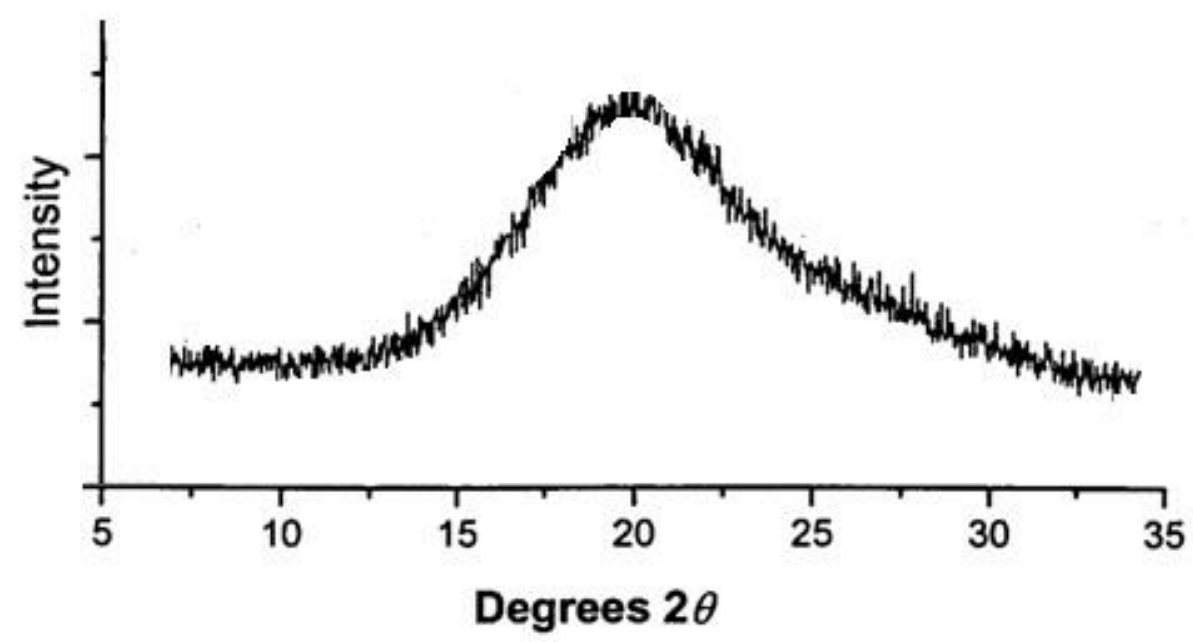

Using the modified Scherer's equation for amorphous polymers,

$$
\mathrm{D}_{\mathrm{ms}}=\mathrm{K} \lambda /\left(\mathrm{B} \cos \theta_{\mathrm{o}}\right)
$$

an average size of ordered mesomorphous clusters of NCD was calculated $\mathrm{D}_{\mathrm{ms}}$ $=1.9 \mathrm{~nm}$ (Ioelovich et al., 2002); whereas the average Bragg's distance between planes in such mesomorphous cluster was $\mathrm{d}_{\mathrm{ms}}=0.45 \mathrm{~nm}$ :

$$
\mathrm{d}_{\mathrm{ms}}=\lambda /\left(2 \sin \theta_{\mathrm{o}}\right)
$$

where $\lambda=0.15418 \mathrm{~nm}$; B is corrected integral width of amorphous scattering in radians; $\mathrm{K}$ equals 1.8 and is the shape factor; $\theta_{\mathrm{o}}$ is the diffraction angle corresponding to maximum of amorphous scattering.

Further, the function of radial distribution of electron density, $F$, was also calculated for precision of the cluster structure:

where $\mathrm{i}(\mathrm{s})$ is the corrected reduced intensity; $\mathrm{s}=(4 \pi / \lambda) \sin \theta$.

$$
F=2 r / \pi \int \operatorname{si}(s) \sin (r s) d s
$$

The $F$-function has five maximums of electron density at distances $r$ of $0.1 ; 0.45 ; 0.9 ; 1.4$ and $1.9 \mathrm{~nm}$ (Ioelovich et al., 2002). The first maximum of $F$ distribution at $0.1 \mathrm{~nm}$ is intramolecular and relates to superposition of lengths between atoms in GPU. Other maximums of electron density are intermolecular and relate to average distance $d_{m s}=0.45 \mathrm{~nm}$ between layers in the mesomorphous cluster, while the third and fourth maximums - to the same distance $d_{m s}$ increased two and three times. The radius of electron density at 1.9 $\mathrm{nm}$ is correlated with an average size of the cluster $\mathrm{D}_{\mathrm{ms}}$. As is follows from calculations, the cluster consists of about five GPUs. These clusters serve as 
centers of growth of crystallites at the recrystallization process of amorphized cellulose (Ioelovich et al., 1982; Ioelovich, 2016a; Paes et al., 2010).

As a result of investigations, a model of mesomorphous cluster was proposed (Ioelovich et al., 2002; Ioelovich, 2014). This cluster has a unit cell with parameters $\boldsymbol{a}$ and $\boldsymbol{b}$ of about $0.9 \mathrm{~nm}, \boldsymbol{c}=1.034 \mathrm{~nm}$ and $\boldsymbol{\gamma}$-angle about $120^{\circ}$ (Figure 6).

Figure 6. Projection of Mesomorphous Unit Cell of the Clusters

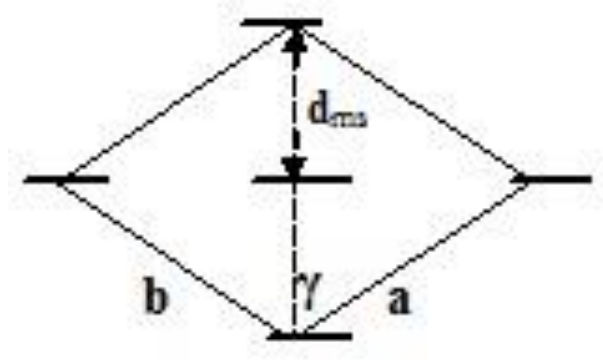

Since only one distance between the layers in the cluster is nearly constant: $\mathrm{d}_{\mathrm{ms}}=0.45 \mathrm{~nm}$, one maximum at $2 \theta \approx 20^{\circ}$ appears on the X-ray diffractogram of amorphous cellulose. The calculated specific gravity of the mesomorphous cluster is $1.48-1.50 \mathrm{~g} / \mathrm{cm}^{3}$, and the average specific gravity of NCD or the amorphous cellulose is $1.45 \mathrm{~g} / \mathrm{cm}^{3}$ (Table 2).

Information about hydrogen bonds in NCD can be obtained using the method of FTIR spectroscopy in combination with deuteration. It was found that after deuteration of cellulose, stretching vibrations of OD-groups in NCD appeared in the range $2300-2680 \mathrm{~cm}^{-1}$ with its maximum at $2500 \mathrm{~cm}^{-1}$. The energy of H-bonds can be calculated from OD-frequency by the equation (Zhbankov et al., 1983):

$$
\mathrm{E}_{\mathrm{H}}=\mathrm{E}_{\mathrm{o}}+\mathrm{K} \sqrt{\Delta v}
$$

where $\mathrm{E}_{\mathrm{o}} \approx 8 \mathrm{~kJ} /$ mole is the minimum base energy; $\mathrm{K}=0.81$.

As a result it was discovered that the energy of H-bonds in NCD varies from 8 to $25 \mathrm{~kJ} / \mathrm{mole}$ (Table 2).

Table 2. Structural Characteristics of Non-crystalline Domains of Cellulose*

\begin{tabular}{|l|c|c|c|c|c|}
\hline Cluster & WP & $\mathbf{d}, \mathrm{g} / \mathrm{cm}^{3}$ & $\mathbf{V}, \mathrm{cm}^{3} / \mathrm{mole}$ & $\begin{array}{c}\mathbf{E}_{\mathbf{H}}, \\
\mathrm{kJ} / \mathrm{mole}\end{array}$ & Transitions \\
\hline DMC & 0.6 & $1.48-1.50$ & $108-110$ & $23-25$ & $\begin{array}{c}\boldsymbol{\alpha}_{1} \text { glass } \\
\text { transition }\end{array}$ \\
\hline MAC & 0.3 & $1.41-1.43$ & $113-115$ & $16-18$ & $\begin{array}{c}\boldsymbol{\alpha}_{2} \text { glass } \\
\text { transition }\end{array}$ \\
\hline LAC & 0.1 & $1.29-1.31$ & $124-126$ & $8-10$ & $\boldsymbol{\beta}$ transition \\
\hline Bulk NCD & 1 & 1.45 & 112 & 20 & $\boldsymbol{\alpha}_{\mathbf{1}}, \boldsymbol{\alpha}_{2}$ and $\boldsymbol{\beta}$ \\
\hline
\end{tabular}

*WP is estimated weight part of a cluster; $\mathbf{d}$ is specific gravity; $\mathbf{V}$ is molar volume

Thus, the detailed studies showed that NCDs of cellulose have heterogeneous structural organization, which consists of dense mesomorphous clusters (DMC), medium packed amorphous clusters (MAC) and loose packed 
amorphous clusters (LAC). The consequence of structural heterogeneity of the NCD of cellulose is the presence of three isophase transitions, where the $\boldsymbol{\alpha}_{1}$ and $\boldsymbol{\alpha}_{2}$ transitions are caused by the occurrence of segmental mobility in dense mesomorphous and medium packed amorphous clusters, respectively; whereas the $\boldsymbol{\beta}$ transition is related to the mobility of GPUs or small segments in loose packed amorphous clusters, which probably are located on the outer surface of nanofibrils (Ioelovich, 2014).

\section{Effect of Plasticizers on Isophase Transitions of Cellulose}

Plasticization of cellulose causes a decrease of the temperatures of $\boldsymbol{\alpha}_{1}, \boldsymbol{\alpha}_{2}$ and $\boldsymbol{\beta}$ transitions (Kaimin et al., 1979). The most widespread and active plasticizer of cellulose is water. Therefore, it is advisable to test the effect of this plasticizer on the relaxation transitions in NCD of cellulose. Unfortunately, water is an easily volatile substance, so its plasticizing effect can be studied only at moderate and low temperatures. As a result, a lowering of the $\boldsymbol{\beta}$ transition temperature under the effect of water was found (Kaimin et al., 1973; Kaimin et al., 1979; Montes et al., 1997; Stratton, 1973). The activation energy of the $\boldsymbol{\beta}$ relaxation involves an entropy contribution that varies with the water content (Stratton, 1973). However, the effect of water on temperatures of $\boldsymbol{\alpha}_{1}$ and $\boldsymbol{\alpha}_{2}$ transitions can be detected only at a high content of the plasticizer close to saturation point (Ioelovich et al., 1979).

Main investigations were devoted to the influence of water on the glass transition temperature of cellulose. It has been shown that at high relative humidity from 80 to $100 \%$ the temperature of glass transition $\left(\mathrm{T}_{\mathrm{g}}\right)$ for moist cellulose is located below room temperature (Ioelovich, 2014; Ioelovich, 2016; Szczesniak et al., 2008). To determine the theoretical $\mathrm{T}_{\mathrm{g}}$ of the cellulose-water system in the wide range of water content, several calculation methods were proposed. For this purpose, Salmen et al., 1977 used Kaelbe's equation (Kaelbe, 1971):

$$
\mathrm{T}_{\mathrm{g}}=\left[\mathrm{T}_{\mathrm{g} 2} \mathrm{X}_{2}+\left(\mathrm{h}_{1} / \mathrm{h}_{2}\right) \mathrm{T}_{\mathrm{g} 1} \mathrm{X}_{1}\right] /\left[\mathrm{X}_{2}+\left(\mathrm{h}_{1} / \mathrm{h}_{1}\right) \mathrm{X}_{1}\right]
$$

where $T_{\mathrm{g} 1}$ and $\mathrm{T}_{\mathrm{g} 2}$ are glass transition temperatures of water and amorphous polymer; $X_{1}$ and $X_{2}$ are mole fraction of water and polymer; while $h_{1}$ and $h_{2}$ are constants of water and polymer, respectively.

Paes with co-authors (Paes et al., 2010) succeeded to obtain the $T_{g}$ of amorphous cellulose (AC) with a certain moisture content, and calculate the dependence of $\mathrm{T}_{\mathrm{g}}$ on moisture content by Couchman's equation (Couchman et al., 1978):

$$
\mathrm{T}_{\mathrm{g}}=\left(\mathrm{W}_{1} \Delta \mathrm{C}_{1} \mathrm{~T}_{\mathrm{g} 1}+\mathrm{W}_{2} \Delta \mathrm{C}_{2} \mathrm{~T}_{\mathrm{g} 2}\right) /\left(\mathrm{W}_{1} \Delta \mathrm{C}_{1}+\mathrm{W}_{2} \Delta \mathrm{C}_{2}\right)
$$

where $T_{\mathrm{g} 1}$ and $\mathrm{T}_{\mathrm{g} 2}$ are glass transition temperatures of water and amorphous cellulose (AC); $\mathrm{W}_{1}$ and $\mathrm{W}_{2}$ are weight fractions of water and $\mathrm{AC} ; \Delta \mathrm{C}_{1}$ and $\Delta \mathrm{C}_{2}$ are change in heat capacity of water at $\mathrm{T}_{\mathrm{g} 1}$ and $\mathrm{AC}$ at $\mathrm{T}_{\mathrm{g} 2}$, respectively.

In our investigations, we used the Fox equation (Fox, 1956): 


$$
\mathrm{T}_{\mathrm{g}}^{-1}=\mathrm{W}_{1} \mathrm{~T}_{\mathrm{g} 1}^{-1}+\mathrm{W}_{2} \mathrm{~T}_{\mathrm{g} 2}{ }^{-1}
$$

where $\mathrm{T}_{\mathrm{g} 1}$ and $\mathrm{T}_{\mathrm{g} 2}$ are glass transitions temperatures of water and amorphous cellulose (AC); $\mathrm{W}_{1}$ and $\mathrm{W}_{2}$ are weight fractions of water and $\mathrm{AC}$, respectively.

The results showed that calculations by means of all three equations (5-7) gave the equal values of theoretical glass transition temperature for wet amorphous cellulose, assuming that the glass transition temperature of dry polymer is $493 \mathrm{~K}$ and for the water is $136 \mathrm{~K}$ (Figure 7).

Figure 7. Theoretical Dependence of Glass Transition Temperature on Weight Part of Water in Amorphous Cellulose or in NCD Calculated by Eq. (5-7)

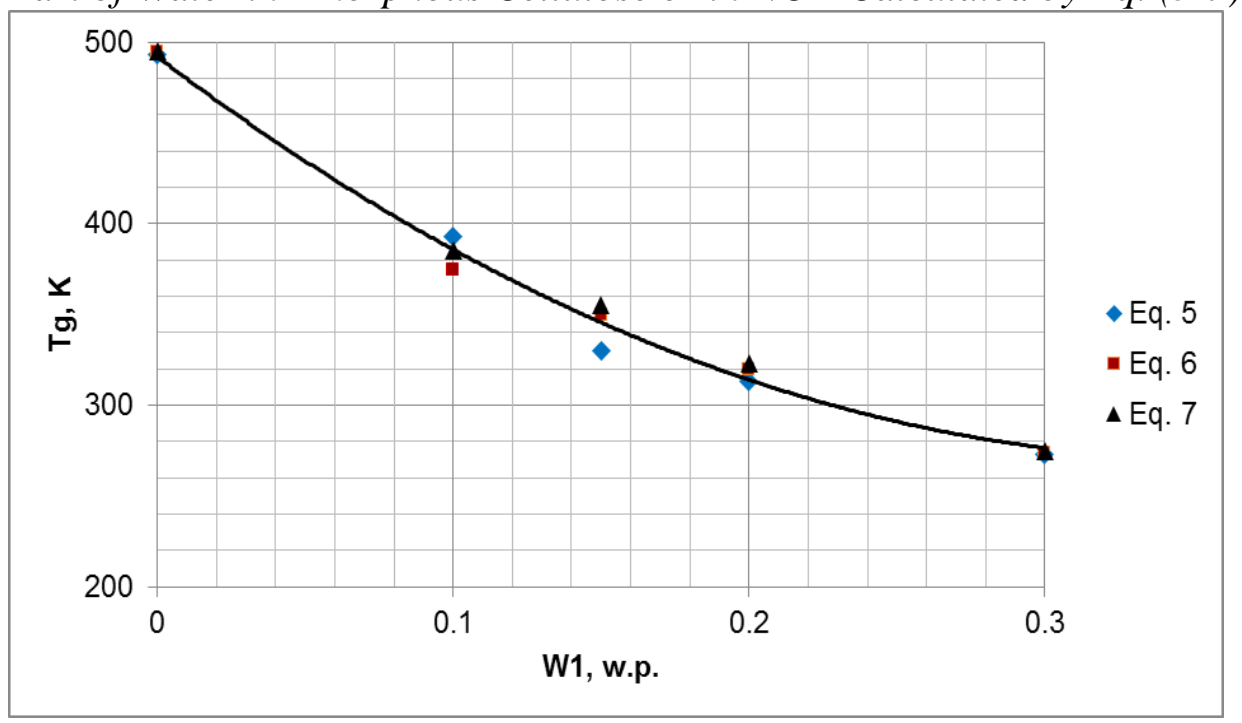

A serious problem arises regarding the extrapolation of the experimental values of $\mathrm{T}_{\mathrm{g}}$ for the wet samples to the value corresponding to dry cellulose. In Figure 8, we tried to reproduce the experimental values of $\mathrm{T}_{\mathrm{g}}$ obtained for wet cellulose samples in some papers (Batzer et al., 1981; Salmen et al., 1977; Szczesniak et al., 2008; Ogiwara et al., 1970). As can be seen from Figure 8, all the experimental $\mathrm{T}_{\mathrm{g}}$ are obtained in the temperature range -50 to $80^{\circ} \mathrm{C}(223$ $353 \mathrm{~K}$ ), i.e. far off $220-230^{\circ} \mathrm{C}(493-503 \mathrm{~K})$, attributed to the temperature of primary $\boldsymbol{\alpha}_{1}$ glass transition of dry cellulose.

To compare the results of various wet cellulose samples obtained in the papers (Batzer et al., 1981; Salmen et al., 1977; Szczesniak et al., 2008; Ogiwara et al., 1970) the moisture content $\left(\mathrm{W}_{\mathrm{NCD}}\right)$ in non-crystalline domains (NCD) of cellulose was calculated (Ioelovich $2016 \mathrm{c}$ ); then $\mathrm{T}_{\mathrm{g}, \mathrm{c}}(\mathrm{K})$ of dry cellulose was calculated from the equation:

$$
\mathrm{T}_{\mathrm{g}, \mathrm{c}}^{-1}=\left[\mathrm{T}_{\mathrm{g}, \mathrm{s}}\left(1-\mathrm{W}_{\mathrm{NCD}}\right)\right]^{-1}-\left[\mathrm{T}_{\mathrm{g}, \mathrm{w}}\left(1-\mathrm{W}_{\mathrm{NCD}}\right) /\left(\mathrm{W}_{\mathrm{NCD}}\right)\right]^{-1}
$$

where $T_{g, s}$ is experimental glass transition temperature of wet sample; $T_{g, w}=136$ $\mathrm{K}$ is the glass transitions temperature of water; $\mathrm{W}_{\mathrm{NCD}}$ is the weight fraction of water in NCD.

The calculations showed that the real $\mathrm{T}_{\mathrm{g}, \mathrm{c}}$ of dry cellulose is about $408 \mathrm{~K}$, i.e. it is the temperature of secondary $\boldsymbol{\alpha}_{2}$ glass transition of dry cellulose (Table 3 ). 
Figure 8. Experimental Values of $T_{g}$ of Wet Cellulose Samples with Different Crystallinity Indexes (CrI)

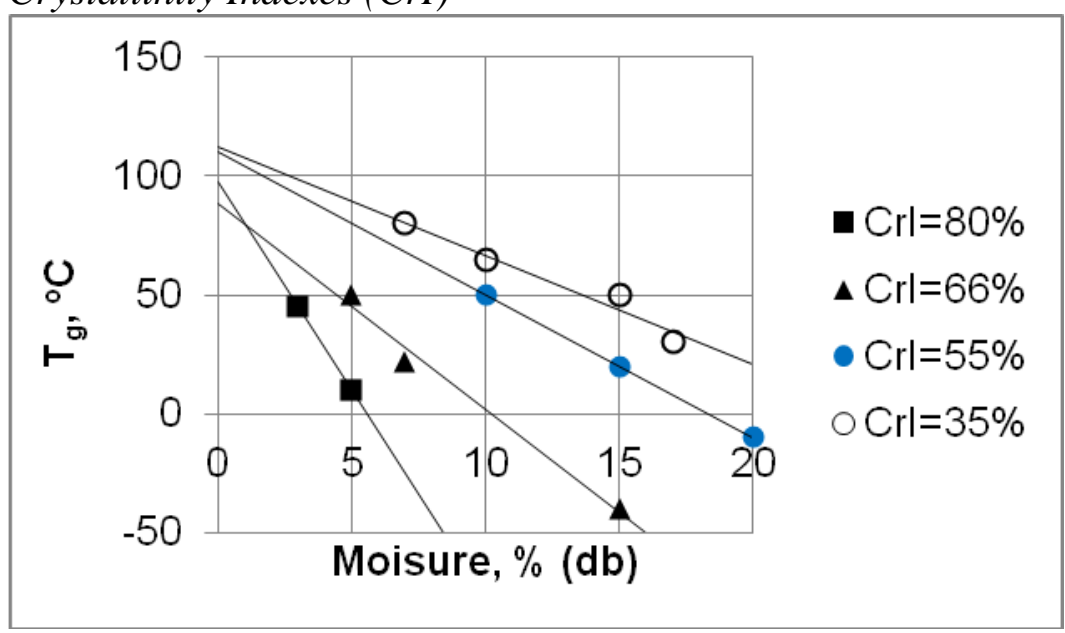

Table 3. Calculated Glass Transition Temperature $\left(T_{g, c}\right)$ of Dry Cellulose

\begin{tabular}{|c|c|c|}
\hline $\mathbf{W}_{\mathrm{NCD}}$, w.p. & Exp. $\mathbf{T}_{\mathrm{g}, \mathrm{s}}, \mathbf{K}$ & Calc. $\mathbf{T}_{\mathrm{g}, \mathrm{c}}, \mathbf{K}$ \\
\hline 0.1 & 345 & 416 \\
\hline 0.2 & 300 & 420 \\
\hline 0.3 & 255 & 408 \\
\hline 0.35 & 235 & 386 \\
\hline & & Average: $\mathbf{4 0 8}$ \\
\hline
\end{tabular}

Note: Exp. $\mathbf{T}_{\mathrm{g}, \mathrm{s}}$ is experimentally obtained glass temperatures of wet samples (Batzer et al., 1981; Salmen et al., 1977; Szczesniak et al., 2008; Ogiwara et al., 1970); Calc. $\mathbf{T}_{\mathrm{g}, \mathrm{c}}$, is calculated glass temperature of dry cellulose.

Using this result, we performed also the reverse calculation $\mathrm{T}_{\mathrm{g}, \mathrm{s}}=\mathrm{f}\left(\mathrm{W}_{\mathrm{NCD}}\right)$, shown in Figure 9.

Figure 9. Experimental and Calculated Values of $T_{g, s}$ of Wet Cellulose Samples

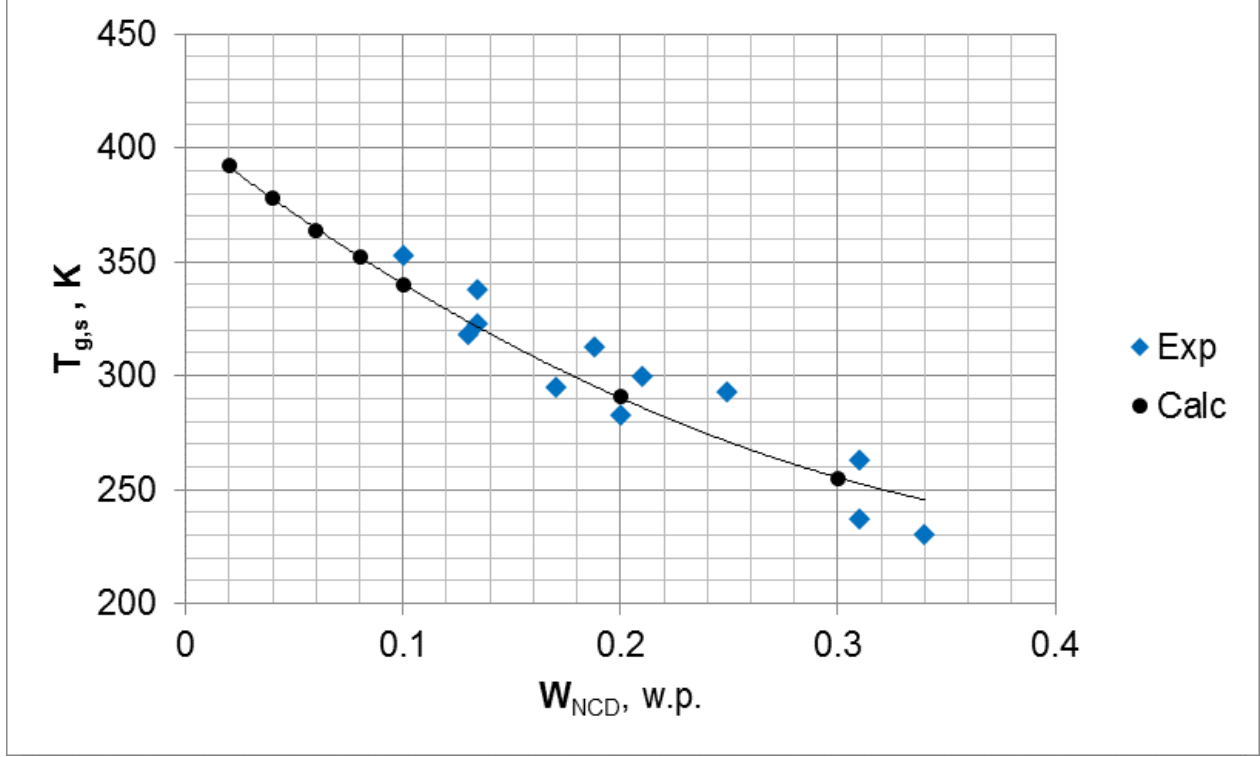


As it follows from Figure 9, in fact the experimental data presented in the papers (Batzer et al., 1981; Salmen et al., 1977; Szczesniak et al., 2008; Ogiwara et al., 1970) describes the dependence of $\boldsymbol{\alpha}_{2}$ glass transition temperature on the water content, i.e. $T_{g, \alpha 2}=f(W)$, instead of $T_{g, \alpha 1}=f(W)$.

Additional difficulties are connected with crystallization of the amorphous cellulose under the action of water, or water and temperature (Ioelovich et al., 1982; Ioelovich, 2016 b; Paes et al., 2010), which distorts the dependence of glass temperature on the water content $\left(\mathrm{W}_{1}\right)$. Furthermore, even for the noncrystallizable semicrystalline cellulose the dependence $T_{g}=f\left(W_{1}\right)$ cannot be obtained at temperatures more than 313-323 K due to water evaporation from the wet cellulose at elevated temperatures.

Some techniques allow the experiments to be conducted in sealed ampoules filled with aqueous solutions of high boiling liquid (e.g. lowmolecular PEG), thus the evaporation of water from the sample can be avoided (Ioelovich et al., 1979). This technique allows the study of both the $\boldsymbol{\alpha}_{1}$ and $\boldsymbol{\alpha}_{2}$ glass transitions, and also the $\boldsymbol{\beta}$ transition, in the cellulose-water system (Ioelovich et al., 1979; Kaimin et al., 1979). These studies have revealed that with increase in the water content in NCD, temperatures of all three transitions decrease (Figure 10).

Figure 10. Dependences of Transition Temperatures on Weight Part of Water in NCD

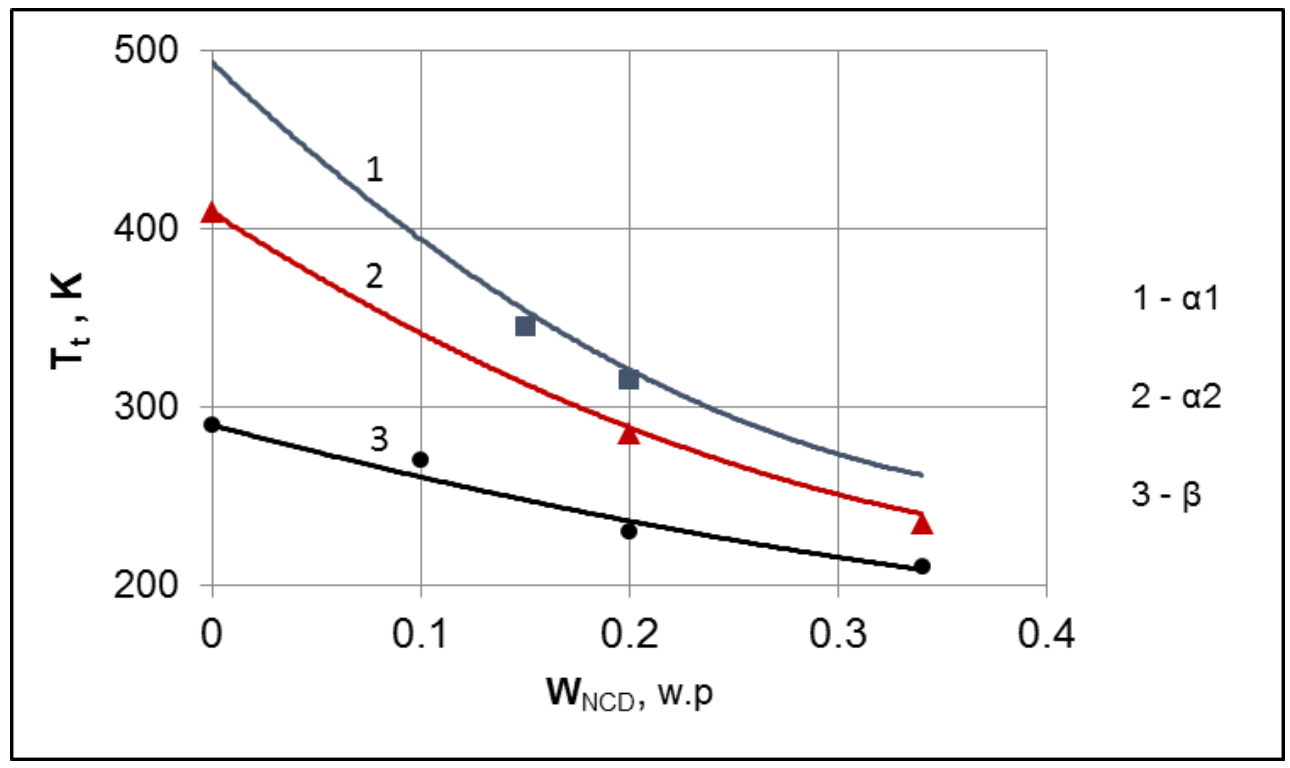

Another problem is that for cellulose samples with various crystallinities, different dependences of the transition temperature on the water content are observed. To get the same results for various cellulose materials it is advisable to use the relative humidity $(\mathrm{RH})$ instead of water content in cellulose (Figure $11)$. 
Figure 11. Dependences of Transition Temperatures $\left(T_{t}\right)$ in Cellulose-Water System on Relative Humidity (RH)

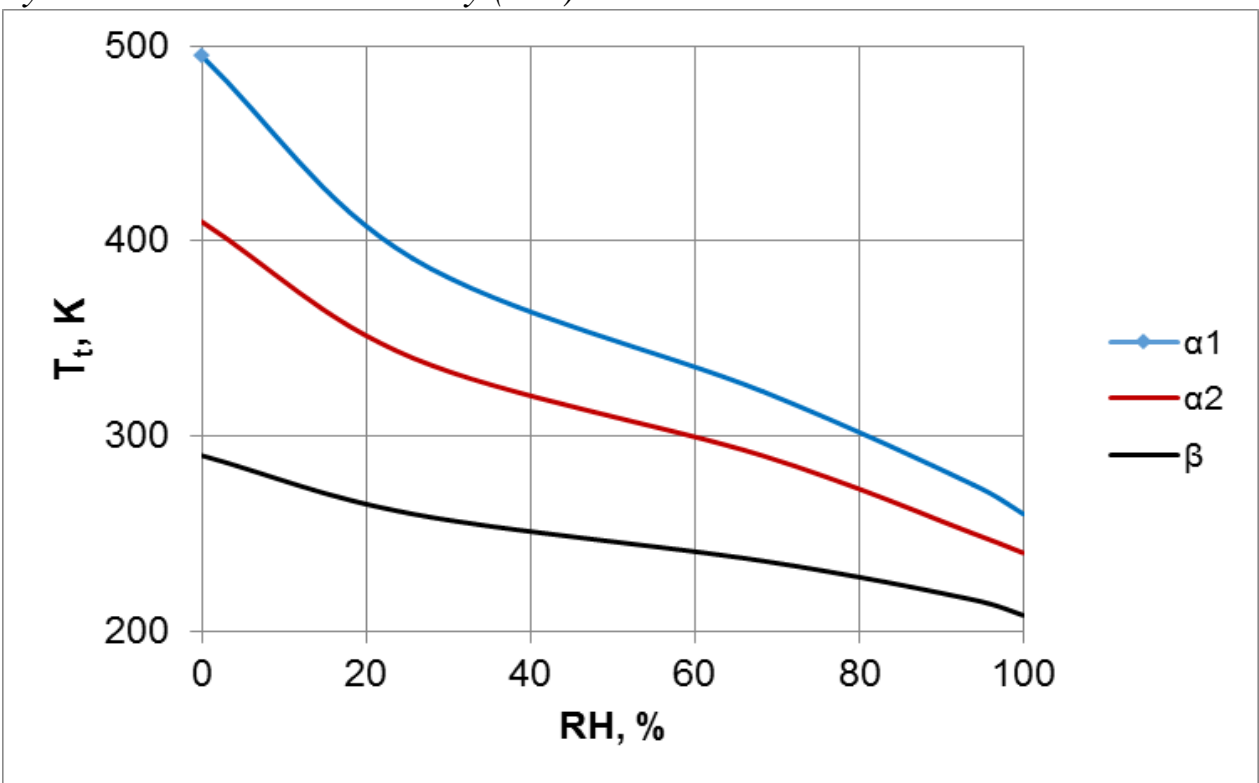

Like water, other polar liquids such as TEPAO, monoethanolamin, ethylenediamine, ethylene glycol, glycerol, and some other polar substances are also plasticizers of cellulose and cause a reduction in temperatures of $\boldsymbol{\alpha}_{1}, \boldsymbol{\alpha}_{2}$ and $\boldsymbol{\beta}$ transitions (Ioelovich et al., 1979; Kaimin et al., 1979). On the contrary, the temperature of the $\gamma$ transition increases with the moistening of cellulose (Roig et al., 2011).

\section{Conclusions}

It has been shown that due to structural heterogeneity the NCD cellulose has three isophase temperature transitions, where the $\boldsymbol{\alpha}_{1}$ transition, $490-500 \mathrm{~K}$, and $\alpha_{2}$ transition, $490-410 \mathrm{~K}$, are caused by the occurrence of segmental mobility in dense mesomorphous and medium packed amorphous clusters, respectively; whereas the $\boldsymbol{\beta}$ transition, $280-300 \mathrm{~K}$, is related to the mobility of small segments in loose packed amorphous clusters, which probably are located on the outer surface of nanofibrils. Under the action of water and other plasticizers, the $\boldsymbol{\alpha}_{1}, \boldsymbol{\alpha}_{2}$ and $\boldsymbol{\beta}$ isophase transitions are shifted to lower temperatures. On the contrary, the temperature of the $\gamma$ transition increases with the moistening of cellulose samples.

\section{References}

Alftha, E., Deruvo, A., and Brown, W. 1973. Glass transition temperature of oligosaccharides. Polymer, 14, 329-330. 
Aziz, K., and Shinouda, H. G. 1973. Acid hydrolysis of cotton cellulose and its thermal transition. Cell. Chem. Technol., 7, 4, 465-478.

Back, E., and Didriksson, E. 1969. Four secondary and glass transition temperature of cellulose evaluated by sonic pulse technique. Svensk Papperstidn., 72, 21, 687694.

Bartenev, G., Aliguliev, R., and Chiteeva, D. 1981. Relaxation transitions in polyethylene. J. Polym. Sci., Part A. 23, 9, 2003-2011.

Batzer, H., and Kreibich, U. T. 1981. Influence of water on thermal transitions in natural polymers and synthetic polyamides. Polym. Bulletin, 5, 11, 585-590.

Borisova, T., Pertopavlovsky, G., and Kotelnikova, N. 1979. Study of temperature transitions in cellulose by dielectric method. J. Polym. Sci., Part A. 21, 9, 20312037.

Boyer, R. F. 1967. Transition and Relaxation in Polymers. Wiley \& Sons, New York.

Couchman, P.R., and Karasz, F.E. 1978. A classical thermodynamic discussion of the effect of composition on glass-transition temperatures. Macromolecules, 11, 117119.

Fainberg, E., and N. Michailov, N 1967. Study of temperature dependence of heat capacity of cellulose fibers. J. Polym. Sci., Part A. 9, 4, 920-926.

Fakirov, S., and Krasteva, B. 2000. On the glass transition temperature of polyethylene as revealed by micro-hardness measurements. J. Macromol. Sci., 39 B, 2, 297-301.

Fox, T. G. 1956. Influence of diluent and copolymer composition on the glass temperature of a polymer system. Bull. Am. Phys. Soc., 1, 123-125.

Ioelovich, M., and Kaimin, I. 1979. Study of temperature transitions of cellulose in liquid media. J. Polym. Sci., Part B. 21, 8, 621-625.

Ioelovich, M., Kaimin, I., and Veveris, G. 1982. The crystallization of amorphized cellulose. J. Polym. Sci., Part A. 24, 6, 1382-1387.

Ioelovich, M., and Laka, M. 2002. Structural analysis of disordered cellulose. SITA, 4, 90-93.

Ioelovich, M. 2014. Cellulose Nanostructured Natural Polymer. LAP, Saarbrücken.

Ioelovich, M. 2016. Models of supramolecular structure and properties of cellulose. $J$. Polym. Sci., Part. A. 58, 1-21.

Ioelovich, M. 2016 a. Peculiarity of phase transitions of cellulose nanocrystallites. ChemXpress, 9, 1-14.

Ioelovich, M. 2016 b. Study of thermodynamic properties of various allomorphs of cellulose. ChemXpress, 9, 259-265.

Ioelovich, M. 2016c. Physicochemical methods for determination of cellulose crystallinity. SITA, 18, 35-42.

Kaelbe, K. 1971. Physical Chemistry of Adhesion. Wiley-Intersci., New York.

Kaimin, I., and Ioelovich, M. 1973. Effect of moisture on transition temperatures of cellulose. J. Polym. Sci., Part B. 15, 10, 764-767.

Kaimin, I., Karlivan, V., and Ioelovich, M. 1979. Temperature transitions of cellulose and their dependence on low molecular substances. News Latvian Acad. Sci., 8, 112-123.

Kargin, V. A., Kozlov, P. V., and Naj-Tchan, W. 1960. On the glass transition temperature in cellulose. Proceed. Acad. Sci. 130, 2, 356-358.

Klason, C., and Kubat, J. 1976. Thermal transition in cellulose. Svensk Papperstidn., $79,15,494-500$.

Klemm, D., Heublein, B., Fink, H-P., and Bohn, A. 2005. Cellulose: fascinating biopolymer and sustainable raw material. Angew. Chem. 44, 2 - 37. 
Manabe, S. Iwata, M., and Kamide, K. 1986. Dynamic mechanical absorptions observed for regenerated cellulose solids in the temperature-range from $280 \mathrm{~K}$ to 600 K. Polym. J., 18, 1, 1-14.

Michailov, G., Artuhov, A., and Borisova, T. 1967. On peculiarities of relaxation of hydroxyl groups in cellulose at low temperatures. J. Polym. Sci., Part B. 9, 2, 138-141.

Mochalov, A. N., Chlustova, T. B., Ioelovich, M. Ya., and Kaimin, I. F. 1982. Effect of crystallinity degree on heat capacity of cellulose. Wood Chem., 4, 66-68.

Montes, H., Mazeau, K., and Cavaille, J. Y. 1977. Secondary mechanical relaxations in amorphous cellulose. Macromolecules, 30, 6977- 6984.

Nordin, S., Nyren, J., and Back, E. 1973. Note on molten cellulose produced in a laser beam. Svensk Papperstidn., 76, 16, 609-610 (1973).

Ogiwara, Y., Kubota, H., Hayashi, S., and Mitomo, N. 1970. Temperature dependency of bound water of cellulose studied by a high-resolution NMR spectrometer. $J$. Appl. Polym. Sci., 14, 2, 303-309.

Paes, S. S., Sun, S., Mac Naughtan, W., Ibbett, R., Ganster, J., Foster, T.J., and Mitchell, J. R. 2010. The glass transition and crystallization of ball milled cellulose. Cellulose, 17, 693-709.

Plotnikov, O., Michailov, A., and Rayavee, E. 1977. Study of extremely slow molecular motions in cellulose by EPR method. J. Polym. Sci., Part A. 19, 11, 2528-2537.

Ramiah, M. V., and Goring, D. A. I. 1965. The thermal expansion of cellulose, hemicellulose and lignin. J. Polym. Sci. Polym. Symp., 11, 27-48.

Roig, F., Dantras, E., Dandurand, J., and Lacabanne, C. 2011. Influence of hydrogen bonds on glass transition and dielectric relaxations of cellulose. J. Appl. Physics, $44,4,1-9$.

Salmen, N. L., and Back, E. L. 1977. The influence of water on the glass phase transition temperature of cellulose. TAPPI, 60, 12, 137-140.

Shen, M., and Eisenberg, A. 1970. Glass transitions in polymers. Rubber Chem. Technol. 43, 95-100.

Stratton, R. A. 1973. Dependence of the viscoelastic properties of cellulose on water content. J Polym. Sci. Polym Chem., 11, 535-544.

Szczesniak, L., Rachocki, A., and Tritt-Goc, J. 2008. Glass transition temperature and thermal decomposition of cellulose powder. Cellulose, 15, 445-451.

Togawa, E., and T. Kondo, T. 2007. Unique structural characteristics of nematic ordered cellulose - stability in water and its facile transformation. J. Polym. Sci. Polym. Phys., 45, 2850-2859.

Van Krevelen, D. W. 1972. Properties of Polymers. Correlation with Chemical Structure. Elsevier Publ., Amsterdam, London, New York.

Wickholm, K., Hult, E. L., Larsson, P. T., Iversen, T., and Lennholm, H. 2001. Quantification of cellulose forms in complex cellulose materials: a chemometric model. Cellulose, 8, 139-148.

Zelenev, Yu., and Glazkov, V. 1972. Relaxation processes in cellulose and its derivatives. J. Polym. Sci., Part A. 14, 1, 16-22.

Zhbankov, R. G., and Kozlov, P. V. 1983. Physics of Cellulose. Science and Technique. 